Formatif: Jurnal Ilmiah Pendidikan MIPA

Vol. 8, No. 1, April 2018, pp. 69-80

p-ISSN: 2088-351X

e-ISSN: 2502-5457

DOI: http://dx.doi.org/10.30998/formatif.v8i1.2351

\title{
A Comparative Study of Three Indonesian Islands on The Performance in Mathematics
}

\author{
Devi Anggriyani \\ Educational Research and Evaluation Department, Yogyakarta State University, \\ Indonesia \\ Martin Iryayo (*) \\ University of Rwanda, Rwanda \\ Educational Research and Evaluation Department, Yogyakarta State University, \\ Indonesia \\ Harun Rasyid \\ Educational Research and Evaluation Department, Yogyakarta State University, \\ Indonesia
}

Received: March 15, 2018 Revised:

Accepted: April 14, 2018

\begin{abstract}
The level of performance among Indonesian students in Mathematics is still critical. This study aims at comparing provinces within each of three islands, and then all three Islands in general, the comparison focused on the performance in mathematics during 2014/2015 national examination. Islands involved in the current research are Java, Sumatera, and Kalimantan. For each province from every island, the researchers selected urban districts by using formula of Krecie \& Morgan (1970), with .05 degree of accuracy, and then systematic sampling to select schools. On Java Island, the researchers selected 669 out of 1065 schools, 508 out of 621 schools on Sumatera Island, and 203 out of 235 schools on Kalimantan Island. Gathering data involved documentation. The researchers analyzed the data by using NCSS 11 and JASP 0.8.3.1. The findings indicated a significant difference among provinces and even Islands on the performance in Mathematics, $\mathrm{p}<.01$, and Sumatera is the most performing island in Mathematics.
\end{abstract}

Keywords: Comparing, Degree of Accuracy, Performance, Rural and Urban Schools

(*) Corresponding Author: $\quad$ iryayomartin2014@gmail.com,

How to Cite: Anggriyani, D., Iryayo, M., and Rasyid, H. (2018). A comparative study of three Indonesian islands on the performance in mathematics. Formatif: Jurnal Ilmiah Pendidikan MIPA, 8 (1): 69-80. http://dx.doi.org/10.30998/formatif.v8i1.2351

\section{INTRODUCTION}

Mathematics has been considered a compulsory subject in the elementary, junior and senior high schools. Leonard (2012) stated that mathematics is one of the important subjects and an indicator of success is student learning. This However, there are always problems raised by the way Mathematics is taught, this can be indicated by the ever-low achievement of students in Mathematics on almost every examination, including the final year national examination conducted by the government. There was revision of 1994 curriculum, but school do not have information yet about how this revision is affecting students' performance in Mathematics (Hadi \& Plomp, 2001). The government has 
Formatif: Jurnal Ilmiah Pendidikan MIPA

Vol. 8, No. 1, April 2018, pp. 69-80

p-ISSN: 2088-351X

e-ISSN: 2502-5457

DOI: http://dx.doi.org/10.30998/formatif.v8i1.2351

revised the curriculum three times so far, but students' performance in mathematics is still low comparing to other countries in the region.

Realistic Mathematics Education (RME) is expected to be a promising teaching approach that meets the Indonesians need in order to improve the teaching of Mathematics. With RME concept, Mathematics seems to be a human activity and should be linked to things that are real (Hadi \& Plomp, 2001). Therefore, it is necessary to explore whether RME is a suited approach to solve problems that frequently occur in Mathematics education and there should be partnership among citizens.

Parents should be concerned with the issue of academic performance of their children. This is the reason why the child's future career can be ensured through his/her academic performance of today. Inheritance and the environment of school have been considered. Governments' distribution of social amenities, such as electricity, water, hospital and educational institutions, always relies on the factors discussed before. It is a common knowledge that many of these social amenities are hugely concentrated in urban areas. These amenities sometimes act as a factor that pulls the educated and rich families to the urban areas. The inappropriate environment and lack of infrastructure hugely contribute to unsuccessful teaching which can lead to poor academic performance (Wilkins \& Ma, 2002).

Students' performances in mathematics can also be dependent on the location of his/her school. As it is believed by many, students from rural schools mostly receive an education that is inferior comparing to their counterparts in the urban localities. Because urban students are provided with better quality in education. They have many facilities and advantages in their education compared to students from rural schools (Faisal \& Mateen, 2016). Faisal \& Mateen (2016) compared performance in academic activities of the rural and urban students; there was significant difference between both groups in terms of their academic performances.

Adepoju, T. L., \& Oluchukwu (2011) conducted a study in Oyo state of Nigeria from 2005 to 2007. The result revealed the remarkable difference between the students from both localities, with remarkable mean scores that urban students obtain rather than rural students. Similarly, another study was undertaken in Pakistan to evaluate the comparative academic performance of rural and urban students at undergraduate level. Result showed that overall performance of urban students was better than rural ones (Onoyase, 2015). All in all, all the findings by the researchers in this paragraph support the remarkable difference between urban and rural localities students' performance.

According to Crane (2010), the goals of schooling are countless, and including not only academic objectives, but also social ones as well. Some authors consider performance in mathematics a better indicator for school effects because they think that it is less influenced by the background of family and home than other subjects (Heyneman, 2005). Having a solid mathematical background helps students develop sophisticated perspective and provides more career alternatives and opportunities.

Qualification of teachers is another factor that impacts on the performance of students in mathematics (Indonesia). Upgrading teaching qualification of teachers, based on 2005 Teacher Law (bachelor degree (S1 required), has resulted in big number of unqualified teachers especially at elementary level. There are a short number of both mathematics and science teachers, particularly in remote areas, who do not have a bachelor degree in mathematics or science concentrations because there is lack of mathematics and science majors in most of local institutions are responsible for training teachers (Hendayana, Asep, \& Imansyah, 2010). For the case of qualification, the question that can be asked about quality education in Indonesian schools is: "is there a 
Formatif: Jurnal Ilmiah Pendidikan MIPA

Vol. 8, No. 1, April 2018, pp. 69-80

p-ISSN: 2088-351X

e-ISSN: 2502-5457

DOI: http://dx.doi.org/10.30998/formatif.v8i1.2351

significant improvement on students' performance in mathematics as long as many teachers are bachelor degrees graduates now?"

There is another dangerous factor that can harm and undermine students' performance, not only in mathematics but also in other subjects in general. The more a teacher is absent from his/her job, the less students' performance is. The table below contains information about teachers' absenteeism across three islands of Indonesia.

Table 1. Teacher Absence by Region (2015)

\begin{tabular}{lcc}
\hline \multicolumn{1}{c}{ Region (Island) } & Absence Rate (\%) & SE \\
\hline Kalimantan & 14.1 & 1.6 \\
\hline Java & 9.1 & 1.7 \\
\hline Sumatera & 8.4 & 1.7 \\
\hline \multicolumn{2}{c}{ Source: Analytical, E.S (2015) }
\end{tabular}

Based on table 1 information, Kalimantan is the most affected island of the three. This means that this absence in Kalimantan must result in students' low performance in mathematics.

TIMSS is an international study that aims at comparing its country-members on their performance in both mathematics and science. Its study in 2007 indicated that Indonesia is number 36 for mathematics (out of 48 countries). What Indonesia needs today is to work hard in mathematics and science in order to pass over other countries in the region because still now Indonesia is classified in the least dominating countries.

The current study wants to compare the urban schools from each province of the three islands and then compare the three islands (Kalimantan, Sumatera, and Java). In the same study, the most and least performing provinces and islands were determined.

\section{METHOD}

The researchers in this survey used the data from Puspendik 2015 about National examination. Only mathematics scores for the target schools located on each island were recorded. The study involved 1380 junior high schools from three Indonesian islands; 203 schools from Kalimantan, 508 schools from Sumatera, and 669 schools from Java Island. Purposive sampling was used to choose public urban schools. Thereafter, systematic sampling was used to determine the sample size after looking for the matching number of schools from the Research Advisors (2006). The latter was used because the population was known; 235 junior high schools from Kalimantan, 537 junior high schools from Sumatera, and 1065 junior high schools from Java. To analyze the data, one way ANOVA was chosen because the study is comparing the mean and there is only one dependent variable. The statistical hypothesis of this study is mentioned below in detail:

1. Provinces
a. Kalimantan
$\mathrm{H}_{0}: \mu_{1=} \mu_{2}=\mu_{3}=\mu_{4}=\mu_{5}$
$\mathrm{H}_{\mathrm{a}}$ : At least one group has observations that tend to be greater than those of the other groups
b. Sumatera
$\mathrm{H}_{0}: \mu_{1=} \mu_{2=} \mu_{3=} \mu_{4=} \mu_{5=} \mu_{6=} \mu_{7=} \mu_{8}=\mu_{9=} \mu_{10}$
$\mathrm{H}_{\mathrm{a}}$ : At least one group has observations that tend to be greater than those of the other groups
c. Java
$\mathrm{H}_{0}: \mu_{1=} \mu_{2}=\mu_{3}=\mu_{4}=\mu_{5}=\mu_{6}$ 
Formatif: Jurnal Ilmiah Pendidikan MIPA

Vol. 8, No. 1, April 2018, pp. 69-80

p-ISSN: 2088-351X

e-ISSN: 2502-5457

DOI: http://dx.doi.org/10.30998/formatif.v8i1.2351

$\mathrm{H}_{\mathrm{a}}$ : At least one group has observations that tend to be greater than those of the other groups

2. All The Three Islands

$\mathrm{H}_{0}: \mu_{1=\mu_{2}=\mu_{3}}$

$\mathrm{H}_{\mathrm{a}}: \mu_{1 \neq} \mu_{2 \neq} \mu_{3}$ or $\mu_{1=\mu_{2 \neq}} \mu_{3}$ or $\mu_{1 \neq} \mu_{2}=\mu_{3}$

\section{FINDINGS AND DISCUSSION}

As the purpose of paper makes it clear, the findings and discussion are organized from descriptive statistics accompanied with plot, ANOVA, and Post-Hoc. For descriptive statistics, the researchers put focus on the central tendencies, mean and median. For ANOVA, the researchers tested the hypothesis ( $p$-value $<\alpha=.01)$. For central tendencies, the researchers stressed more on the highest and lowest mean and median, sometimes effect size. For Post-Hoc comparison, three computational techniques were adapted; Tukey, Scheffe, and Bonfenie. Finally, the plots were graphed for supporting what descriptive statistics and Post-Hoc comparison revealed.

Table 2. Descriptive Statistics for Kalimantan Island

\begin{tabular}{lcccccc}
\hline \multicolumn{1}{c}{ Group } & Count & Mean & Effect & Median & $\begin{array}{c}\text { Standard } \\
\text { Deviation }\end{array}$ & $\begin{array}{c}\text { Error } \\
\text { (MSE/ni) }\end{array}$ \\
\hline All & 203 & 52.71 & 51.37 & & & \\
\hline $\begin{array}{l}\text { A: } \\
\text { Kalimantan }\end{array}$ & & & & & & \\
\hline $\begin{array}{l}\text { Central } \\
\text { Kalimantan }\end{array}$ & 29 & 47.81 & -3.55 & 47.19 & 13.34 & 1.923 \\
\hline $\begin{array}{l}\text { East } \\
\text { Kalimantan }\end{array}$ & 73 & 44.46 & -6.91 & 41.82 & 8.293 & 1.212 \\
$\begin{array}{l}\text { North } \\
\text { Kalimantan }\end{array}$ & 11 & 39.69 & -11.68 & 36.72 & 8.979 & 3.122 \\
\hline $\begin{array}{l}\text { South } \\
\text { Kalimantan }\end{array}$ & 49 & 64.02 & 12.66 & 67.5 & 10.27 & 1.479 \\
\hline $\begin{array}{l}\text { West } \\
\text { Kalimantan }\end{array}$ & 41 & 60.85 & 9.483 & 64.39 & 11.65 & 1.617 \\
\hline
\end{tabular}

Table 2 contains the information about the highest and lowest mean score on Mathematics across all the provinces of Kalimantan. The highest mean and median with yellow color (64.02 and 67.5) show the province whose junior high schools have the most performing students on mathematics. In contrast, the lowest mean and median with red color (39.69 and 36.72) indicate different information.

Table 3. One-Way ANOVA for Kalimantan Island

\begin{tabular}{lccccc}
\hline Cases & $\begin{array}{c}\text { Sum of } \\
\text { Squares }\end{array}$ & df & $\begin{array}{c}\text { Mean } \\
\text { Square }\end{array}$ & F & p \\
\hline Province_Kalimantan & 16523 & 4 & 4130.8 & $38.52<.001$ \\
\hline Residual & 21232 & 198 & 107.2 & \\
\hline
\end{tabular}

The information that can be read from Table 3, the hypothesis testing supports the rejection of $\mathrm{H}_{0}$ because $p<.01$, it means at least one province in Kalimantan has schools whose students' performance on mathematics differ from that of students schooling in other provinces. Therefore, there is a significant difference among students' mathematics performances all over the island. 
Formatif: Jurnal Ilmiah Pendidikan MIPA

Vol. 8, No. 1, April 2018, pp. 69-80

p-ISSN: 2088-351X

e-ISSN: 2502-5457

DOI: http://dx.doi.org/10.30998/formatif.v8i1.2351

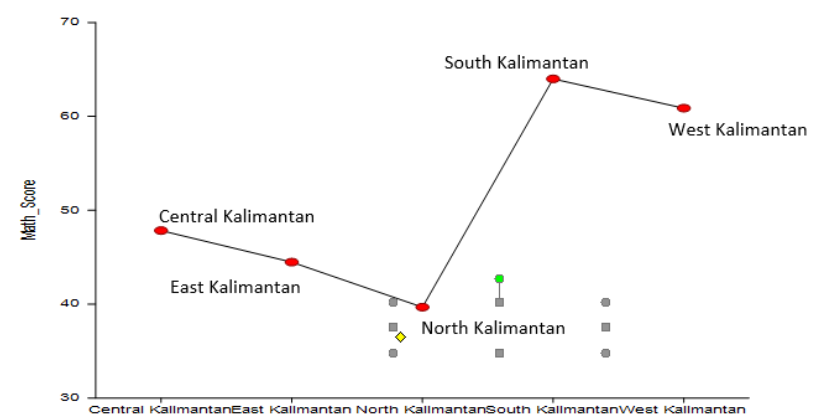

Graph 1. Means Plot of Math Score of Kalimantan Island

The plot illustrates the ordering of provinces in Kalimantan Island based on the mean score in mathematics. South Kalimantan is the most performing province while North Kalimantan is the least performing one.

Table 4. Post Hoc Comparisons-Province for Kalimantan Island

\begin{tabular}{|c|c|c|c|c|c|c|c|}
\hline & & $\begin{array}{c}\text { Mean } \\
\text { Difference }\end{array}$ & SE & t & $\mathbf{p}_{\text {tukey }}$ & $\mathbf{p}_{\text {scheffe }}$ & e $p$ bonf \\
\hline \multirow[t]{4}{*}{$\begin{array}{l}\text { Central } \\
\text { Kalimantan } \\
\end{array}$} & $\begin{array}{l}\text { East } \\
\text { Kalimantan }\end{array}$ & 3.358 & 2.273 & 1.477 & 0.566 & 0.702 & 1.000 \\
\hline & $\begin{array}{l}\text { North } \\
\text { Kalimantan }\end{array}$ & 8.127 & 3.667 & 2.216 & 0.169 & 0.300 & 0.278 \\
\hline & $\begin{array}{l}\text { South } \\
\text { Kalimantan }\end{array}$ & -16.211 & 2.426 & -6.682 & $<.001$ & $<.001$ & $<.001$ \\
\hline & $\begin{array}{l}\text { West } \\
\text { Kalimantan }\end{array}$ & -13.036 & 2.513 & -5.188 & $<.001$ & $<.001$ & $<.001$ \\
\hline \multirow[t]{3}{*}{ East Kalimantan } & $\begin{array}{l}\text { North } \\
\text { Kalimantan }\end{array}$ & 4.769 & 3.349 & 1.424 & 0.601 & 0.731 & 1.000 \\
\hline & $\begin{array}{l}\text { South } \\
\text { Kalimantan }\end{array}$ & -19.569 & 1.912 & $\begin{array}{l} \\
10.232\end{array}$ & $<.001$ & $<.001$ & $<.001$ \\
\hline & $\begin{array}{l}\text { West } \\
\text { Kalimantan }\end{array}$ & -16.394 & 2.021 & -8.112 & $<.001$ & $<.001$ & $<.001$ \\
\hline \multirow[t]{2}{*}{$\begin{array}{l}\text { North } \\
\text { Kalimantan } \\
\end{array}$} & $\begin{array}{l}\text { South } \\
\text { Kalimantan }\end{array}$ & -24.337 & 3.455 & -7.044 & $<.001$ & $<.001$ & $<.001$ \\
\hline & $\begin{array}{l}\text { West } \\
\text { Kalimantan }\end{array}$ & -21.162 & 3.516 & -6.019 & $<.001$ & $<.001$ & $<.001$ \\
\hline $\begin{array}{l}\text { South } \\
\text { Kalimantan }\end{array}$ & $\begin{array}{l}\text { West } \\
\text { Kalimantan }\end{array}$ & 3.175 & 2.192 & 1.449 & 0.585 & 0.718 & 1.000 \\
\hline
\end{tabular}

To read Table 4, we look at mean difference. In the table, the highest positive mean difference is between Central and North Kalimantan (8.127). The highest negative mean difference is between North and South Kalimantan (-24.337).

Table 5. Descriptive Statistics for Sumatera Island

\begin{tabular}{lllllll}
\hline Group & Count & Mean & Effect & $\begin{array}{l}\text { Medi } \\
\text { an }\end{array}$ & $\begin{array}{l}\text { Standard } \\
\text { Deviation }\end{array}$ & $\begin{array}{l}\text { Error } \\
\text { (MSE/ni) }\end{array}$ \\
\hline All & 508 & 64.244 & 59.885 & & & \\
\hline $\begin{array}{l}\text { B: } \\
\text { Sumatera }\end{array}$ & & & & & & \\
\hline Aceh & 69 & 76.72 & 16.84 & 79.92 & 12.71 & 1.736 \\
\hline $\begin{array}{l}\text { Bangka } \\
\text { Belitung }\end{array}$ & 11 & 46.48 & -13.41 & 40.95 & 12.50 & 4.347 \\
\hline Bengkulu & 27 & 42.21 & -17.68 & 38.84 & 9.835 & 2.775 \\
\hline Jambi & 41 & 77.62 & 17.74 & 83.62 & 13.28 & 2.252 \\
\hline $\begin{array}{l}\text { Kepulaua } \\
\text { n Riau }\end{array}$ & 10 & 46.72 & -13.17 & 40.62 & 13.83 & 4.559 \\
\hline Lampung & 46 & 58.47 & -1.42 & 56.3 & 12.26 & 2.126 \\
\hline $\begin{array}{l}\text { North } \\
\text { Sumatera }\end{array}$ & 110 & 68.42 & 8.538 & 73.97 & 14.54 & 1.375 \\
\hline Riau & 53 & 68.51 & 8.621 & 75.04 & 15.17 & 1.980 \\
\hline $\begin{array}{l}\text { South } \\
\text { Sumatera }\end{array}$ & 56 & 50.72 & -9.166 & 42.07 & 16.63 & 1.927 \\
\hline $\begin{array}{l}\text { West } \\
\text { Sumatera }\end{array}$ & 85 & 62.995 & 3.111 & 63.06 & 16.44 & 1.564 \\
\hline & & & & & & \\
\hline
\end{tabular}


Formatif: Jurnal Ilmiah Pendidikan MIPA

Vol. 8, No. 1, April 2018, pp. 69-80

p-ISSN: 2088-351X

e-ISSN: 2502-5457

DOI: http://dx.doi.org/10.30998/formatif.v8i1.2351

Table 5 contains the information about the highest and lowest mean score on Mathematics across all the provinces of Sumatera. The highest mean and median with yellow color (77.62 and 83.62) shows the province whose junior high schools have the most performing students on mathematics. In contrast, the lowest mean and median with red color (42.21 and 38.84) denote different information.

Table 6. One-Way ANOVA for Sumatera Island

\begin{tabular}{lccccc}
\hline \multicolumn{1}{c}{ Cases } & $\begin{array}{c}\text { Sum of } \\
\text { Squares }\end{array}$ & df & $\begin{array}{c}\text { Mean } \\
\text { Square }\end{array}$ & F & p \\
\hline Province_Sumatera & 52534 & 9 & 5837.1 & $28.08<.001$ \\
\hline Residual & 103517 & 498 & 207.9 & \\
\hline
\end{tabular}

Table 6 is about testing the hypothesis concerning performances on Math along all provinces of Sumatera. The hypothesis testing supports the rejection of $\mathrm{H}_{0}$ because $p<$ .01 , it means at least one province on Sumatera has schools whose students' performance on mathematics differ from that of students schooling in other provinces. Therefore, the comparison of mean proves a significant difference in terms of students' performance in mathematics for all junior high schools located in the provinces involved in this study.

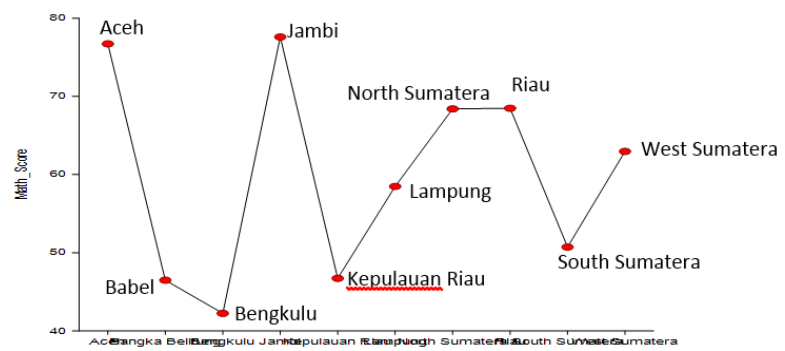

Graph 2. Means Plot of Math Score of Sumatera Island

If we look at the plot drawn above with naked eye, there is interesting information. The mean score on Mathematics in Aceh and Jambi is not proving the significant difference. The same case appears for North Sumatera and Riau, and Bangka Belitung and Kepulauan Riau.

Table 7. Post Hoc Comparisons - Provinces for Sumatera Island

\begin{tabular}{|c|c|c|c|c|c|c|c|}
\hline & & $\begin{array}{c}\text { Mean } \\
\text { Difference }\end{array}$ & SE & $\mathbf{t}$ & $\mathbf{p}_{\text {tukey }}$ & $\mathbf{p}_{\text {scheffe }}$ & $\mathbf{p}_{\text {bonf }}$ \\
\hline \multirow[t]{9}{*}{ Aceh } & $\begin{array}{l}\text { Bangka } \\
\text { Belitung }\end{array}$ & 30.243 & 4.681 & 6.461 & $<.001$ & $<.001$ & $<.001$ \\
\hline & Bengkulu & 34.517 & 3.273 & 10.547 & $<.001$ & $<.001$ & $<.001$ \\
\hline & Jambi & -0.902 & 2.843 & -0.317 & 1.000 & 1.000 & 1.000 \\
\hline & $\begin{array}{l}\text { Kepulauan } \\
\text { Riau }\end{array}$ & 30.004 & 4.878 & 6.150 & $<.001$ & $<.001$ & $<.001$ \\
\hline & Lampung & 18.253 & 2.744 & 6.651 & $<.001$ & $<.001$ & $<.001$ \\
\hline & $\begin{array}{l}\text { North } \\
\text { Sumatera }\end{array}$ & 8.297 & 2.214 & 3.747 & 0.006 & 0.124 & 0.009 \\
\hline & Riau & 8.214 & 2.633 & 3.119 & 0.053 & 0.375 & 0.086 \\
\hline & $\begin{array}{l}\text { South } \\
\text { Sumatera }\end{array}$ & 26.001 & 2.593 & 10.027 & $<.001$ & $<.001$ & $<.001$ \\
\hline & $\begin{array}{l}\text { West } \\
\text { Sumatera }\end{array}$ & 13.725 & 2.336 & 5.875 & $<.001$ & $<.001$ & $<.001$ \\
\hline
\end{tabular}


Formatif: Jurnal Ilmiah Pendidikan MIPA

Vol. 8, No. 1, April 2018, pp. 69-80

p-ISSN: 2088-351X

e-ISSN: 2502-5457

DOI: http://dx.doi.org/10.30998/formatif.v8i1.2351

\begin{tabular}{|c|c|c|c|c|c|c|c|}
\hline & & $\begin{array}{c}\text { Mean } \\
\text { Difference }\end{array}$ & SE & $\mathbf{t}$ & P tukey & $\mathbf{p}_{\text {scheffe }}$ & $\mathbf{p}_{\text {bonf }}$ \\
\hline \multirow[t]{8}{*}{$\begin{array}{l}\text { Bangka } \\
\text { Belitung } \\
\end{array}$} & Bengkulu & 4.274 & 5.157 & 0.829 & 0.998 & 1.000 & 1.000 \\
\hline & Jambi & -31.145 & 4.896 & -6.362 & $<.001$ & $<.001$ & $<.001$ \\
\hline & $\begin{array}{l}\text { Kepulauan } \\
\text { Riau }\end{array}$ & -0.239 & 6.299 & -0.038 & 1.000 & 1.000 & 1.000 \\
\hline & Lampung & -11.990 & 4.839 & -2.478 & 0.260 & 0.725 & 0.610 \\
\hline & $\begin{array}{l}\text { North } \\
\text { Sumatera }\end{array}$ & -21.946 & 4.559 & -4.813 & $<.001$ & 0.007 & $<.001$ \\
\hline & Riau & -22.028 & 4.777 & -4.611 & $<.001$ & 0.013 & $<.001$ \\
\hline & $\begin{array}{l}\text { South } \\
\text { Sumatera }\end{array}$ & -4.242 & 4.755 & -0.892 & 0.996 & 1.000 & 1.000 \\
\hline & $\begin{array}{l}\text { West } \\
\text { Sumatera }\end{array}$ & -16.518 & 4.620 & -3.576 & 0.012 & 0.176 & 0.017 \\
\hline \multirow[t]{7}{*}{ Bengkulu } & Jambi & -35.419 & 3.573 & -9.912 & $<.001$ & $<.001$ & $<.001$ \\
\hline & $\begin{array}{l}\text { Kepulauan } \\
\text { Riau }\end{array}$ & -4.513 & 5.337 & -0.846 & 0.997 & 1.000 & 1.000 \\
\hline & Lampung & -16.264 & 3.495 & -4.653 & $<.001$ & 0.011 & $<.001$ \\
\hline & $\begin{array}{l}\text { North } \\
\text { Sumatera }\end{array}$ & -26.220 & 3.097 & -8.468 & $<.001$ & $<.001$ & $<.001$ \\
\hline & Riau & -26.303 & 3.409 & -7.716 & $<.001$ & $<.001$ & $<.001$ \\
\hline & $\begin{array}{l}\text { South } \\
\text { Sumatera }\end{array}$ & -8.516 & 3.378 & -2.521 & 0.238 & 0.703 & 0.540 \\
\hline & $\begin{array}{l}\text { West } \\
\text { Sumatera }\end{array}$ & -20.792 & 3.185 & -6.528 & $<.001$ & $<.001$ & $<.001$ \\
\hline \multirow[t]{6}{*}{ Jambi } & $\begin{array}{l}\text { Kepulauan } \\
\text { Riau }\end{array}$ & 30.906 & 5.085 & 6.078 & $<.001$ & $<.001$ & $<.001$ \\
\hline & Lampung & 19.155 & 3.097 & 6.186 & $<.001$ & $<.001$ & $<.001$ \\
\hline & $\begin{array}{l}\text { North } \\
\text { Sumatera }\end{array}$ & 9.200 & 2.638 & 3.487 & 0.016 & 0.208 & 0.024 \\
\hline & Riau & 9.117 & 2.999 & 3.040 & 0.067 & 0.417 & 0.112 \\
\hline & $\begin{array}{l}\text { South } \\
\text { Sumatera }\end{array}$ & 26.903 & 2.963 & 9.079 & $<.001$ & $<.001$ & $<.001$ \\
\hline & $\begin{array}{l}\text { West } \\
\text { Sumatera }\end{array}$ & 14.627 & 2.741 & 5.336 & $<.001$ & $<.001$ & $<.001$ \\
\hline \multirow[t]{5}{*}{$\begin{array}{l}\text { Kepulauan } \\
\text { Riau } \\
\end{array}$} & Lampung & -11.751 & 5.030 & -2.336 & 0.342 & 0.792 & 0.895 \\
\hline & $\begin{array}{l}\text { North } \\
\text { Sumatera }\end{array}$ & -21.707 & 4.762 & -4.558 & $<.001$ & 0.015 & $<.001$ \\
\hline & Riau & -21.790 & 4.971 & -4.384 & $<.001$ & 0.025 & $<.001$ \\
\hline & $\begin{array}{l}\text { South } \\
\text { Sumatera }\end{array}$ & -4.003 & 4.950 & -0.809 & 0.998 & 1.000 & 1.000 \\
\hline & $\begin{array}{l}\text { West } \\
\text { Sumatera }\end{array}$ & -16.279 & 4.820 & -3.377 & 0.024 & 0.252 & 0.035 \\
\hline \multirow[t]{4}{*}{ Lampung } & $\begin{array}{l}\text { North } \\
\text { Sumatera }\end{array}$ & -9.956 & 2.532 & -3.933 & 0.003 & 0.082 & 0.004 \\
\hline & Riau & -10.038 & 2.905 & -3.455 & 0.018 & 0.220 & 0.027 \\
\hline & $\begin{array}{l}\text { South } \\
\text { Sumatera }\end{array}$ & 7.748 & 2.869 & 2.701 & 0.160 & 0.607 & 0.322 \\
\hline & $\begin{array}{l}\text { West } \\
\text { Sumatera }\end{array}$ & -4.528 & 2.639 & -1.716 & 0.766 & 0.966 & 1.000 \\
\hline \multirow[t]{3}{*}{ North Sumatera } & Riau & -0.083 & 2.411 & -0.034 & 1.000 & 1.000 & 1.000 \\
\hline & $\begin{array}{l}\text { South } \\
\text { Sumatera }\end{array}$ & 17.704 & 2.367 & 7.480 & $<.001$ & $<.001$ & $<.001$ \\
\hline & $\begin{array}{l}\text { West } \\
\text { Sumatera }\end{array}$ & 5.427 & 2.082 & 2.607 & 0.198 & 0.658 & 0.424 \\
\hline Riau & $\begin{array}{l}\text { South } \\
\text { Sumatera }\end{array}$ & 17.787 & 2.763 & 6.438 & $<.001$ & $<.001$ & $<.001$ \\
\hline
\end{tabular}


Formatif: Jurnal Ilmiah Pendidikan MIPA

Vol. 8, No. 1, April 2018, pp. 69-80

p-ISSN: 2088-351X

e-ISSN: 2502-5457

DOI: http://dx.doi.org/10.30998/formatif.v8i1.2351

\begin{tabular}{cccccccc}
\hline & $\begin{array}{c}\text { Mean } \\
\text { Difference }\end{array}$ & SE & $\mathbf{t}$ & $\mathbf{p}_{\text {tukey }}$ & $\mathbf{p}_{\text {scheffe }}$ & $\mathbf{p}_{\text {bonf }}$ \\
\hline $\begin{array}{l}\text { West } \\
\text { Sumatera }\end{array}$ & 5.510 & 2.523 & 2.184 & 0.442 & 0.853 & 1.000 \\
\hline $\begin{array}{l}\text { West } \\
\text { South Sumatera }\end{array}$ & -12.276 & 2.481 & -4.947 & $<.001$ & 0.004 & $<.001$ \\
\hline \hline
\end{tabular}

In Sumatera, there is a remarkable difference between provinces. The highest positive and negative mean difference is between Aceh-Bengkulu, and Bengkulu-Jambi with 34. 517 and 35.419 respectively. However that, there are some other provinces between which the mean difference is not highly significant e.g. North Sumatera-Riau, Bangka Belitung-Kepualaun Riau and Aceh-Jambi.

Table 8. Descriptive Statistics for Java Island

\begin{tabular}{lllllll}
\hline Group & Count & Mean & Effect & $\begin{array}{l}\text { Media } \\
\text { n }\end{array}$ & $\begin{array}{l}\text { Standard } \\
\text { Deviation }\end{array}$ & $\begin{array}{l}\text { Error } \\
\text { V(MSE/ni) }\end{array}$ \\
\hline All & 669 & 63.209 & 63.99 & & & \\
\hline C: Java & & & & & & \\
\hline Banten & 83 & 50.881 & -13.09 & 45.65 & 16.62487 & 1.390519 \\
\hline $\begin{array}{l}\text { Central } \\
\text { Java }\end{array}$ & 102 & 57.691 & -6.277 & 55.08 & 16.43636 & 1.254341 \\
\hline $\begin{array}{l}\text { DKI } \\
\text { Jakarta }\end{array}$ & 184 & 73.075 & 9.106 & 72.835 & 7.471444 & 0.9339141 \\
\hline East Java & 128 & 67.673 & 3.705 & 69.87 & 14.20396 & 1.119724 \\
\hline $\begin{array}{l}\text { West } \\
\text { Java }\end{array}$ & 155 & 56.410 & -7.559 & 53.41 & 10.82055 & 1.017537 \\
\hline $\begin{array}{l}\text { Yogyaka } \\
\text { rta }\end{array}$ & 17 & 78.079 & 14.111 & 80.09 & 12.70483 & 3.072496 \\
\hline
\end{tabular}

Two rows from table 8 are colored to deliver specific information about mean score, median, and effect size based on mathematics performance on Java Island. The island is known, in the whole country, to be develop in different domains including education. The capital city of Indonesia is located on the same island. Moreover, there are other provinces which host a lot of schools. Coming back to table 8, the province on Java Island whose mean score, median and effect are the highest, is DI Yogyakarta with $78.079,80.09$, and 14.111 respectively. In contrast, Banten Province has the lowest mean, median, and effect scores; 50.881, 45.65, and -13.09 respectively. The Capital city, DKI Jakarta, has most schools comparing to other provinces located on the same island (184 schools), it comes after DI Yogyakarta if we consider the scores for variables stated above.

Table 9. One-Way ANOVA for Java Island

\begin{tabular}{lccccc}
\hline \multicolumn{1}{c}{ Cases } & Sum of Squares & df & Mean Square & F & p \\
\hline Province_Java & 47104 & 5 & 9420.9 & 58.70 & $<.001$ \\
\hline Residual & 106401 & 663 & 160.5 & & \\
\hline
\end{tabular}

Table 9 is just about testing the hypothesis concerning performances on mathematics along all provinces of Java. The hypothesis testing supports the rejection of $\mathrm{H}_{0}$ because $p<.01$, it means at least one province on Java has schools whose students' performance on mathematics differ from that of students schooling in the rest of provinces. Therefore, the comparison of mean proves a significant difference in terms of students' performance in mathematics for all junior high schools based in the provinces involved in this study. 
Formatif: Jurnal Ilmiah Pendidikan MIPA

Vol. 8, No. 1, April 2018, pp. 69-80

p-ISSN: 2088-351X

e-ISSN: 2502-5457

DOI: http://dx.doi.org/10.30998/formatif.v8i1.2351

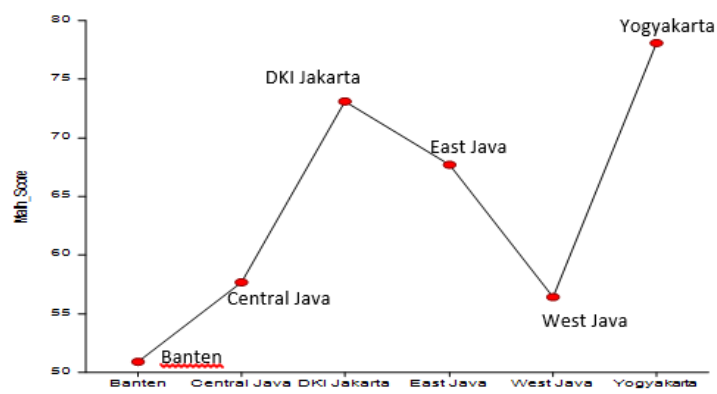

Graph 3. Means Plot of Math Score of Java Island

The plot above confirms the information in table 6. Here, it can be seen that all provinces are different, this can be easily detected by looking at the position of red dots. Yogyakarta comes the first with the highest mean score on mathematics. Banten is in the critical region on the plot because it is not far from the lowest score (50).

Table 10. Post Hoc Comparisons-Provinces of Java Island

\begin{tabular}{|c|c|c|c|c|c|c|c|}
\hline & & $\begin{array}{c}\text { Mean } \\
\text { Difference }\end{array}$ & SE & $\mathbf{t}$ & $\mathbf{p}_{\text {tukey }}$ & p scheffe & $\mathbf{p}$ bonf \\
\hline \multirow[t]{5}{*}{ Banten } & $\begin{array}{l}\text { Central } \\
\text { Java }\end{array}$ & -6.810 & 1.873 & -3.636 & 0.003 & 0.022 & 0.004 \\
\hline & DKI Jakarta & -22.193 & 1.675 & $13.250^{-}$ & $<.001$ & $<.001$ & $<.001$ \\
\hline & East Java & -16.792 & 1.785 & -9.406 & $<.001$ & $<.001$ & $<.001$ \\
\hline & West Java & -5.528 & 1.723 & -3.208 & 0.016 & 0.069 & 0.021 \\
\hline & Yogyakarta & -27.198 & 3.373 & -8.065 & $<.001$ & $<.001$ & $<.001$ \\
\hline \multirow[t]{4}{*}{$\begin{array}{l}\text { Central } \\
\text { Java }\end{array}$} & DKI Jakarta & -15.384 & 1.564 & -9.837 & $<.001$ & $<.001$ & $<.001$ \\
\hline & East Java & -9.982 & 1.681 & -5.937 & $<.001$ & $<.001$ & $<.001$ \\
\hline & West Java & 1.281 & 1.615 & 0.793 & 0.966 & 0.987 & 1.000 \\
\hline & Yogyakarta & -20.388 & 3.319 & -6.144 & $<.001$ & $<.001$ & $<.001$ \\
\hline \multirow[t]{3}{*}{$\begin{array}{l}\text { DKI } \\
\text { Jakarta } \\
\end{array}$} & East Java & 5.401 & 1.458 & 3.704 & 0.003 & 0.018 & 0.003 \\
\hline & West Java & 16.665 & 1.381 & 12.066 & $<.001$ & $<.001$ & $<.001$ \\
\hline & Yogyakarta & -5.005 & 3.211 & -1.559 & 0.608 & 0.787 & 1.000 \\
\hline \multirow[t]{2}{*}{ East Java } & West Java & 11.264 & 1.513 & 7.445 & $<.001$ & $<.001$ & $<.001$ \\
\hline & Yogyakarta & -10.406 & 3.270 & -3.182 & 0.017 & 0.073 & 0.023 \\
\hline West Java & Yogyakarta & -21.670 & 3.237 & -6.695 & $<.001$ & $<.001$ & $<.001$ \\
\hline
\end{tabular}

Across Java Island, the highest positive and negative mean difference is between DKI Jakarta-West Java, and Banten-Yogyakarta with 16.665 and -27.198 respectively. Remembering that when the mean difference is positive the first province mean score on mathematics is higher than its counterpart, e.g. West Java-Yogyakarta, the mean difference equals -21.670. It means West Java has the lower mean score. This technique of interpreting means difference can be applied to other pairs of provinces.

Table 11. Descriptive Statistics for Three Islands of Indonesia

\begin{tabular}{lllllll}
\hline Group & $\begin{array}{l}\text { Cou } \\
\text { nt }\end{array}$ & Mean & Effect & $\begin{array}{l}\text { Medi } \\
\text { an }\end{array}$ & $\begin{array}{l}\text { Standard } \\
\text { Deviation }\end{array}$ & $\begin{array}{l}\text { Error } \\
\text { V(MSE/ni) }\end{array}$ \\
\hline All & 1380 & 62.046 & 60.06 & & & \\
\hline A: Java & 669 & 63.209 & 3.154 & 65.01 & 15.15909 & 0.6140159 \\
\hline $\begin{array}{l}\text { B. } \\
\text { Kalimantan }\end{array}$ & 203 & 52.712 & -7.343 & 50.91 & 13.6714 & 1.114666 \\
\hline $\begin{array}{l}\text { C. } \\
\text { Sumatera }\end{array}$ & 508 & 64.244 & 4.189 & 69.88 & 17.54404 & 0.7046295 \\
\hline
\end{tabular}


Formatif: Jurnal Ilmiah Pendidikan MIPA

Vol. 8, No. 1, April 2018, pp. 69-80

p-ISSN: 2088-351X

e-ISSN: 2502-5457

DOI: http://dx.doi.org/10.30998/formatif.v8i1.2351

Table 11 englobes the information about the highest and lowest mean scores on Mathematics among all three islands; Kalimantan, Sumatera and Java. The island with the highest mean, median and effect scores (yellow) is Sumatera (64.24364, 69.88 and 4.189022 , respectively). The latter depicts the island whose junior high schools have the most performing students on mathematics. In contrast, the lowest mean, median and effect is Kalimantan (red) with 52.71192, 50.91 and -7.342699 respectively.

Table 12. One-Way ANOVA for Three Islands

\begin{tabular}{lccccc}
\hline Cases & Sum of Squares & df & Mean Square & F & p \\
\hline Island & 21044 & 2 & 10521.8 & 41.72 & $<.001$ \\
\hline Residual & 347312 & 1377 & 252.2 & & \\
\hline
\end{tabular}

By comparing mean among the three islands, it was found that the idea of rejecting $\mathrm{H}_{0}$ is worthwhile. The p-value $<.01$, which means that there is a significant difference on mathematics performance by students from all junior high schools located on Java, Sumatera, and Kalimantan islands. This also demonstrates that at least one group has observations that tend to be greater than those of the other groups. We no longer assumed that at least one group but all provinces are different in terms of students' performance in mathematics.

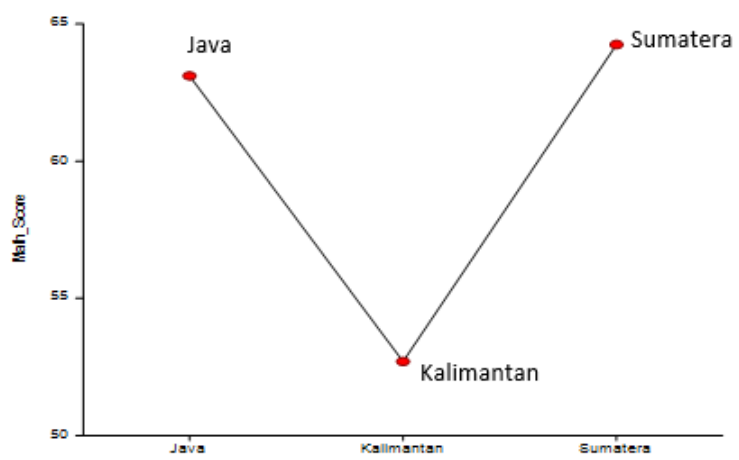

Graph 4. Means Plot of Math Score of Three Indonesian Islands

The graph drawn above explains well how the three islands differ based on mathematics mean score. There is a long distance from dot standing for Kalimantan to the dots for Sumatera and Java. It is clear that the mean score in mathematics for all schools located in Kalimantan varies between 50 and 55. On the other hand, Sumatera and Java are not hugely different because both of them are above 60. In brief, Kalimantan is still far to reach the other two islands.

Table 13. Post Hoc Comparisons for the Three Islands

\begin{tabular}{|c|c|c|c|c|c|c|c|}
\hline & & Mean Difference & $\mathbf{S E}$ & $\mathbf{t}$ & $\mathbf{p}$ tukey & $\mathbf{p}_{\text {scheffe }}$ & $\mathbf{p}$ bonf \\
\hline Java & Kalimantan & 10.496 & 1.273 & 8.248 & $<.001$ & $<.001$ & $<.001$ \\
\hline & Sumatera & -1.035 & 0.935 & -1.108 & 0.505 & 0.542 & 0.804 \\
\hline Kalimantan & Sumatera & -11.532 & 1.317 & -8.758 & $<.001$ & $<.001$ & $<.001$ \\
\hline
\end{tabular}

The information from table 13, is not far from what can be read from the plot, but it gives further explanation with numbers. If you look at the mean difference between Java-Kalimantan (10.496) and Kalimantan-Sumatera (-11.532), the difference is not big just -1.035 . Having a look at all p-values in table 13, the differences between Java and 
Formatif: Jurnal Ilmiah Pendidikan MIPA

Vol. 8, No. 1, April 2018, pp. 69-80

p-ISSN: 2088-351X

e-ISSN: 2502-5457

DOI: http://dx.doi.org/10.30998/formatif.v8i1.2351

Sumatera is not significant because $p>.01$. Summarily, Sumatera comes the best in students' performance on mathematics national test 2014/2015.

\section{CONCLUSION}

By concluding this report, it is necessary to look through all the results and achievement of the objectives the research was conducted for. On each island, the schools by province, shows significant difference among them towards their students' performance on mathematics. The latter, was not only found for provinces comparison but also the islands in general. There are some provinces on each island that are showing a good step in promoting outstanding performance in mathematics, e.g. Yogyakarta Province on Java, Jambi Province on Sumatera, and South Kalimantan on Kalimantan Island. However, there are other provinces which are far left behind in performing well on Mathematics test, e.g. Banten Province on Java, Bengkulu Province on Sumatera, and North Kalimantan Province on Kalimantan. It can be recommended that:

1. The government of Indonesia through the Ministry of Education should make empirical and periodic follow up on mentioned Provinces;

2. Javanese junior high schools should revise the teaching strategies because it was found that Sumatera already passed over while people have mind that Java is the most developed in all domains;

3. Central government through the local government should motivate the teachers in order to revise their professionalism;

4. Indonesian schools, especially junior high schools are highly recommended to schedule study tours within and outside the island;

5. All people concerned with education should make sure of quality facilities, collaboration staff-teachers, teacher-student, parent-staff-teacher, and avoiding unreasonable absence.

\section{REFERENCES}

Adepoju, T. L., \& Oluchukwu, E. E. (2011). A study of secondary school students' academic performance at the Senior School Certificate examinations and implications for educational planning and policy in Nigeria. African Research Review, 5(6), 314-333.

Analytical, E. S. (2015). Teacher Absenteeism in Indonesia.

Madya, S. (2011). Teori dan Praktik Penelitian Tindakan (Action Research). Bandung: Alfabeta.

Crane, C. C. (2010). Mathematics performance in public and Catholic elementary schools: Explaining the disparity. University of Illinois at Urbana-Champaign.

Faisal, R., Shinwari, L., \& Mateen, H. (2016). Evaluation of the academic achievement of rural versus urban undergraduate medical students in pharmacology examinations. Asian Pacific Journal of Reproduction, 5(4), 317-320.

Hadi, S., \& Plomp, T. (2001). Introducing Realistic Mathematics Education to Junior High School Mathematics Teachers in Indonesia.

Hendayana, S., Asep, S., \& Imansyah, H. (2010). Indonesia's issues and challenges on quality improvement of mathematics and science education. Journal of International Cooperation in Education, 41-51. 
Formatif: Jurnal Ilmiah Pendidikan MIPA

Vol. 8, No. 1, April 2018, pp. 69-80

p-ISSN: 2088-351X

e-ISSN: 2502-5457

DOI: http://dx.doi.org/10.30998/formatif.v8i1.2351

Heyneman, S. P. (2005). Student background and student achievement: What is the right question? American Journal of Education, 112(1), 1-9.

Ismail, S. F. Z. H., Shahrill, M., \& Mundia, L. (2015). Factors contributing to effective mathematics teaching in secondary schools in Brunei Darussalam. ProcediaSocial and Behavioral Sciences, 186, 474-481.

Khan, B. B., Nawaz, R., Chaudhry, K. M., Hyder, A. U., \& Butt, T. M. (2012). Evaluation of comparative academic performance of undergraduate students at university level. J Anim Plant Sci, 22(3), 798-801.

Leonard. (2012). Level of appreciation, self concept, and positive thinking on mathematics learning achievement. The International Journal of Social Sciences, $6(1): 10-17$.

Onoyase, A. (2015). Stress Coping Strategies among Guidance Counsellors in the Performance of Their Jobs in Secondary Schools.

Owoeye, J. S., \& Yara, P. O. (2011). School location and academic achievement of secondary school in Ekiti State, Nigeria. Asian social science, 7(5), 170.

Wilkins, J. L., \& Ma, X. (2002). Predicting student growth in mathematical content knowledge. The Journal of Educational Research, 95(5), 288-298. 